\title{
Vitamin D levels in systemic sclerosis patients: a meta-analysis
}

\author{
This article was published in the following Dove Press journal: \\ Drug Design, Development and Therapy \\ 27 October 2017 \\ Number of times this article has been viewed
}

\section{Lin An \\ Ming-hui Sun \\ Feng Chen \\ Jin-ran Li}

Department of Dermatology, China-Japan Union Hospital of Jilin University, Changchun, People's Republic of China
Correspondence: Jin-ran Li

Department of Dermatology,

China-Japan Union Hospital of Jilin

University, 126 Xiantai St, Changchun

130033, Jilin Province, People's Republic of China

Tel/fax +8643184641026

Email lijr1990@।63.com
Purpose: This study aimed to investigate the association between vitamin D and systemic sclerosis (SSc) by meta-analysis.

Methods: A comprehensive search was performed through June 12, 2017. Pooled standardized mean difference (SMD) was used to estimate the mean vitamin D difference between case and control groups (or between diffused- and limited-type SSc). Pooled risk ratios (RRs) with 95\% confidence intervals (CIs) were used to assess the impact of vitamin D level on clinical characteristics of SSc patients. All statistical analyses were performed using Revman 5.0 software.

Results: The search yielded six studies with a total of 554 SSc patients and 321 healthy controls. The meta-analysis showed that SSc patients suffered from decreased vitamin D levels ( $\mathrm{SMD}=-8.72 \mathrm{ng} / \mathrm{mL} ; 95 \% \mathrm{CI}$ : -10.11 to -7.32 ) compared with healthy controls. The meta-analysis results of three studies with $240 \mathrm{SSc}$ patients (93 diffused-type and 147 limitedtype SSc patients) showed that diffused-type SSc patients exhibited lower vitamin D levels (SMD $=-4.71 \mathrm{ng} / \mathrm{mL} ; 95 \% \mathrm{CI}:-8.98$ to -0.44 ) compared with limited-type SSc patients. However, vitamin D level was not found to be associated with Rodnan score (SMD =-2.29 ng/mL, 95\% CI: -8.49 to $3.91, P=0.47$ ), systolic pulmonary pressure (SMD $=-1.68 \mathrm{ng} / \mathrm{mL}, 95 \%$ CI: -10.79 to $7.43, P=0.72$ ), gastrointestinal ulcer ( $R R=1.01,95 \% \mathrm{CI}: 0.53-1.93, P=0.98$ ), or pulmonary involvement $(\mathrm{RR}=1.01,95 \% \mathrm{CI}: 0.36-2.86, P=0.99)$ in $\mathrm{SSc}$ patients.

Conclusion: SSc patients exhibited lower vitamin D levels compared with healthy controls. Vitamin D levels in diffused-type SSc patients were significantly lower than those in limitedtype SSc patients. The severity of clinical features was not associated with the extent of vitamin D deficit. Therefore, we hypothesize that SSc patients, especially diffused type, have lower vitamin D levels, and that the decrease of vitamin D levels might not be an accelerating factor of SSc severity.

Keywords: vitamin D, systemic sclerosis, meta-analysis

\section{Introduction}

Over the past decade, it has become clear that the role of vitamin $\mathrm{D}$ goes beyond the modulation of calcium homeostasis and bone health. An important extraskeletal effect of vitamin D is the regulation of the immune system. ${ }^{1}$ Current data link vitamin D status to many autoimmune diseases, such as vitiligo, systemic lupus erythematosus, multiple sclerosis, rheumatoid arthritis and inflammatory bowel disease. ${ }^{2-4}$ This is well explained by the ubiquitous presence of vitamin D receptors which are expressed on natural killer cells, T lymphocytes and B lymphocytes, suggesting an effect on innate and adaptive immune responses. ${ }^{5,6}$ Studies have found that vitamin D deficiency (VDD) impairs immune responses and self-tolerance by compromising the functions of these cells. ${ }^{7}$

Systemic sclerosis (SSc) is a connective tissue disease characterized by the obliteration of the microvasculature, dysfunction of the immune system and excessive 
deposition of matrix substances in the skin and internal organs. ${ }^{8}$ It remains a public health problem globally, disabling normal functions and impairing the quality of life. More than $50 \%$ of SSc patients eventually die as a direct result of the disease. ${ }^{9}$ A large number of factors have been reported which may affect the initiation and progression of SSc; one of these factors is serum vitamin D level.

Several studies have aimed to determine whether VDD is associated with SSc; however, results are inconsistent. There have been three systemic reviews exploring the association between VDD and SSc, which concluded that hypovitaminosis D is very frequent in SSc and severe in a relevant percentage of patients. ${ }^{10-12}$ However, a meta-analysis was neither performed, nor did they perform a further analysis based on factors such as vitamin D insufficiency and VDD.

Therefore, we conducted a meta-analysis to provide additional evidence to support or refute an association between hypovitaminosis D and SSc.

\section{Methods}

\section{Literature search strategy}

We performed a comprehensive search in the databases including PubMed, Web of Science, Cochrane Library and Wanfang through June 12, 2017. The keywords used for searching potentially relevant data were "Vitamin D" and "Systemic sclerosis". The search strategy used in PubMed was "(Vitamin D) AND Systemic sclerosis". In addition, we manually examined reference lists of retrieved articles to further identify potentially relevant publications.

\section{Inclusion and exclusion criteria}

Studies were included if they met the following criteria: 1) clinical studies investigating the role of vitamin $D$ in patients diagnosed with SSc; 2) case-control studies calculating vitamin D levels in SSc patients and healthy patients, or studies investigating the relationship between vitamin $\mathrm{D}$ level and the characteristics of SSc patients; 3) for case-control studies, the mean value and standard deviation of vitamin D levels should be provided in the article, and for other studies, relative data should be provided to assess the impact of vitamin D on SSc patients. Cases, letters, reviews and articles with insufficient data were excluded. If multiple studies were performed on the population of same origin, only the most recent and comprehensive research was used.

\section{Data extraction}

Two authors performed data extraction independently, and any discrepancies were resolved by a consensus. The main characteristics of the included case-control studies were extracted, including the first author, publication time, country, study sample size (case-control group) and mean and standard deviation of vitamin D levels (case-control group). The vitamin D levels in both limited-type and diffused-type SSc patients were also extracted. Other collected data included cutoff values for VDD/vitamin D insufficiency and characteristics of SSc patients (Rodnan score, gastrointestinal ulcer, pulmonary involvement and systolic arterial pressure).

\section{Statistical analysis}

For continuous variables, pooled standardized mean difference (SMD) was used to estimate the mean vitamin D difference between case and control groups (or between diffused-type and limited-type SSc). For discontinuous variables, pooled risk ratios (RRs) with $95 \%$ confidence intervals (CIs) were used to assess the impact of vitamin level on clinical characteristics of SSc patients. Statistical heterogeneity was assessed using the $I^{2}$ test, and $I^{2}>50 \%$ was defined as high heterogeneity. A fixed-effect model was used when $I^{2}$ was $<50 \%$; otherwise, a random-effect model was used. All statistical analyses were performed using Revman 5.0 software. Publication bias was assessed using funnel plots. $P$-value $<0.050$ was considered statistically significant.

\section{Results}

\section{Literature information}

Initially, 609 publications were retrieved from the available databases. After reading titles and abstracts of each, 577 were excluded which included reviews, duplicates, letters, case reports and articles not analyzing the relationship between vitamin D and SSc. An additional 24 studies were excluded because they were not case-control studies or available data could not be extracted. Eight publications met the inclusion criteria and were used in our meta-analysis. The search process for the potential publication literature is shown in Figure 1.

\section{Characteristics of included studies}

As shown in Table 1, six case-control studies were included in this meta-analysis, which investigated vitamin D level differences between SSc patients and healthy controls. ${ }^{13-18}$ The publication dates ranged from 2011 to 2016, and the populations of origin were based in four countries (Italy 3, China 1, Israel 1 and Brazil 1). The study sample size for SSc patients ranged from 10 to 327, and the sample size for healthy controls ranged from 10 to 141 . Four studies reported the duration of disease, and four provided information on SSc types. As shown in Table 2, three studies investigated 


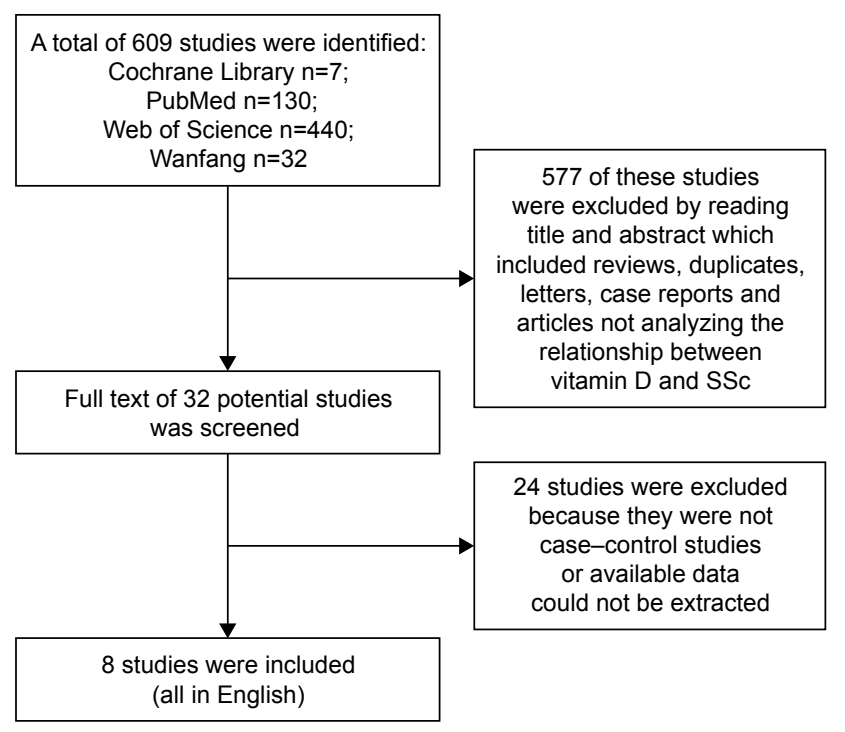

Figure I Flow diagram of study selection in this meta-analysis. Abbreviation: SSc, systemic sclerosis.

the vitamin D level in different types of SSc, ${ }^{15,17,20}$ and three studies investigated the correlation of vitamin D level with clinical outcomes of SSc patients..$^{15,19,20}$

\section{Meta-analysis of the vitamin D difference between case and control groups}

Six studies with a total of 554 SSc patients and 321 healthy controls investigated differences in vitamin $\mathrm{D}$ levels between case and control groups. Since high heterogeneity was not identified between the included studies $\left(I^{2}=50 \%\right)$, a fixedeffect model was used. Meta-analysis results showed that SSc patients suffered from decreased vitamin D levels ( $\mathrm{SMD}=-8.72 \mathrm{ng} / \mathrm{mL} ; 95 \% \mathrm{CI}:-10.11$ to -7.32 ) compared with healthy people (Figure 2).

\section{Meta-analysis of the vitamin D difference between diffused- and limited-type SSc patients}

In total, three studies with $240 \mathrm{SSc}$ patients (93 diffused and 147 limited type) investigated differences in vitamin D levels.
High heterogeneity was identified between the included studies $\left(I^{2}=65 \%\right)$; therefore, a random-effect model was used. Metaanalysis results determined that diffused-type SSc patients exhibited lower vitamin D levels (SMD =-4.71 ng/mL; 95\% CI: -8.98 to -0.44 ) compared with limited-type SSc patients (Figure 3 ).

\section{Meta-analysis of correlation of vitamin D level with clinical characteristics in SSc patients}

According to the three included studies, vitamin D level $\geq 30 \mathrm{ng} / \mathrm{mL}$ was defined as optimal, between 10 and $30 \mathrm{ng} / \mathrm{mL}$ as insufficient and $<10 \mathrm{ng} / \mathrm{mL}$ as deficient. Firstly, data from $166 \mathrm{SSc}$ patients (113 insufficient and 53 deficient SSc patients) were used to investigate the correlation of vitamin D levels with the Rodnan score. Metaanalysis results determined that there was no correlation between vitamin $\mathrm{D}$ level and Rodnan score in SSc patients (SMD $=-2.29 \mathrm{ng} / \mathrm{mL}, 95 \% \mathrm{CI}:-8.49$ to $3.91, P=0.47$; from the random-effect model). Secondly, two studies with 108 SSc patients (77 insufficient and 31 deficient SSc patients) investigated the correlation of vitamin D levels with systolic pulmonary arterial pressure, gastrointestinal ulcers and pulmonary involvement. Meta-analysis results demonstrated that vitamin D level was not associated with systolic pulmonary pressure $(\mathrm{SMD}=-1.68 \mathrm{ng} / \mathrm{mL}, 95 \% \mathrm{CI}:-10.79$ to $7.43, P=0.72$, from random-effect model), gastrointestinal ulcer $(\mathrm{RR}=1.01,95 \% \mathrm{CI}: 0.53-1.93, P=0.98$, from fixed-effect model), or pulmonary involvement $(\mathrm{RR}=1.01$, 95\% CI: 0.36-2.86, $P=0.99$, from random-effect model) (Figure 4A-D).

\section{Publication bias}

In this meta-analysis, we did not use a test to examine potential publication bias. However, no publication bias was found for the vitamin D difference between case and control groups, which was seen from the nearly symmetrical funnel plots for this meta-analysis (Figure 5).

Table I Characteristics of included case-control studies

\begin{tabular}{|c|c|c|c|c|c|c|c|}
\hline Study & $\begin{array}{l}\text { Publication } \\
\text { time }\end{array}$ & Country & $\begin{array}{l}\text { Sample size } \\
\text { (case-control) }\end{array}$ & $\begin{array}{l}\text { Case } \\
(\text { mean } \pm \text { SD })\end{array}$ & $\begin{array}{l}\text { Control } \\
(\text { mean } \pm \text { SD) }\end{array}$ & $\begin{array}{l}\text { Duration of } \\
\text { disease (years) }\end{array}$ & Type of SSc \\
\hline Zhang et $\mathrm{al}^{13}$ & 2015 & China & $60 / 60$ & $26.5 I \pm 6.27$ & $36.29 \pm 14.24$ & $3.72 \pm 3.53$ & NA \\
\hline Shinjo et $\mathrm{al}^{14}$ & 2011 & Brazil & $10 / 10$ & $18.1 \pm 6.4$ & $25.1 \pm 6.6$ & $7.2 \pm 3.5$ & Diffuse/limited: $7 / 3$ \\
\hline Corrado et $\mathrm{al}^{15}$ & 2015 & Italy & $64 / 35$ & $15.68 \pm 10.18$ & $22.93 \pm 9.12$ & $7.3 \pm 2.8 / 8.9 \pm 4.2$ & Diffuse/limited: $31 / 33$ \\
\hline Seriolo et $\mathrm{al}^{16}$ & 2011 & Italy & $53 / 35$ & $21.7 \pm 13.4$ & $39.4 \pm 15.4$ & NA & NA \\
\hline Arnson et $\mathrm{al}^{17}$ & 2011 & Israel & $327 / 141$ & $13.5 \pm 9.0$ & $21.6 \pm 9.7$ & NA & Diffuse/limited: $35 / 90$ \\
\hline Atteritano et $\mathrm{al}^{18}$ & 2016 & Italy & $40 / 40$ & $25.77 \pm 12.84$ & $35.08 \pm 9.07$ & $9.11 \pm 6.74$ & Diffuse/limited: I2/28 \\
\hline
\end{tabular}

Abbreviations: NA, not available; SD, standard deviation; SSc, systemic sclerosis. 
Table 2 Characteristics of included studies investigating the vitamin $\mathrm{D}$ level in different types of systemic sclerosis patients or studies investigating the correlation of vitamin D with the clinical characteristics of SSc patients

\begin{tabular}{|c|c|c|c|c|c|c|}
\hline Study & $\begin{array}{l}\text { Publication } \\
\text { time }\end{array}$ & Country & $\begin{array}{l}\text { Sample size (diffusel } \\
\text { limited type) }\end{array}$ & $\begin{array}{l}\text { Vitamin D level } \\
\text { (diffused/limited type) }\end{array}$ & $\begin{array}{l}\text { Clinical } \\
\text { characteristics }\end{array}$ & Cutoff value for vitamin $D$ \\
\hline Groseanu et $\mathrm{a}^{20}$ & 2016 & Romania & $5 \mathrm{I}(27 / 24)$ & $15.92 \pm 9.1 / 22.05 \pm 10.8$ & $\begin{array}{l}\text { Rodnan score, ulcer, } \\
\text { pulmonary, sPAP }\end{array}$ & $\begin{array}{l}10<\text { insufficiency }<30(n=34) \\
\text { deficiency }<10(n=12)\end{array}$ \\
\hline Corrado et al ${ }^{15}$ & 2015 & Italy & $64(3 \mid / 33)$ & $11.53 \pm 7.46 / 19.22 \pm 11.13$ & Rodnan score & $\begin{array}{l}10<\text { insufficiency }<30(n=36) \\
\text { deficiency }<10(n=22)\end{array}$ \\
\hline Arnson et $\mathrm{al}^{17}$ & 2011 & Israel & $125(35 / 90)$ & $|3.0 \pm 7.0 /| 4.3 \pm|| .2$ & NA & NA \\
\hline Caramaschi et al ${ }^{19}$ & 2010 & Italy & 65 (NA) & NA & $\begin{array}{l}\text { Rodnan score, ulcer, } \\
\text { pulmonary, sPAP }\end{array}$ & $\begin{array}{l}10<\text { insufficiency }<30(n=43) \\
\text { deficiency }<10(n=19)\end{array}$ \\
\hline
\end{tabular}

Note: Vitamin $D$ level data presented as mean \pm SD.

Abbreviations: NA, not available; sPAP, systolic pulmonary artery pressure; SSc, systemic sclerosis.

\section{Discussion}

Vitamin D is a steroid hormone that plays a critical role not only in calcium and bone metabolism but also in immunity. VDD is known to be linked with many autoimmune diseases. ${ }^{2,3}$ Numerous studies have been conducted to study whether vitamin D is associated with SSc; however, they produced varying results. ${ }^{13-20}$ There have been several systemic reviews on associations between vitamin $\mathrm{D}$ and $\mathrm{SSc}$; however, a meta-analysis has not been done, nor has further analysis based on factors such as vitamin D insufficiency and VDD been conducted.

To the best of our knowledge, this is the first meta-analysis investigating the association between serum vitamin $\mathrm{D}$ levels and SSc. The relationship between vitamin D and SSc is a disputed issue, and it is still unclear whether vitamin $\mathrm{D}$ is a cause, accelerating factor or consequence of SSc. Our results demonstrate that SSc patients have decreased vitamin D levels when compared with healthy people. The main source of vitamin D for the body is exposure to sunlight, which induces the conversion of a precursor located in the skin to the active form of vitamin D, and vitamin D status is also affected by nutrient intake as well as gastrointestinal absorption. ${ }^{17}$
Poor vitamin D status in SSc patients seems to be related to skin or mucosa thickening, which results in insufficient sun exposure and insufficient intake and malabsorption. We thus hypothesized that SSc patients, especially diffused type, would have lower vitamin D levels. Further, our study demonstrated that serum vitamin D levels in diffused-type SSc patients were significantly lower than those in limitedtype SSc patients. Therefore, we assume that low vitamin D level is a manifestation of SSc, and that SSc is responsible for VDD.

The association between SSc patients and vitamin D levels might also be explained by the hypothesis that low vitamin D level directly increases the risk of autoimmune disease. Vitamin D has been substantiated to have an antifibrotic effect on fibroblasts, inhibit synthesis of the extracellular matrix and regulate the skin's immune system. ${ }^{21}$ The receptors of vitamin D on immune cells can modulate immune responses. ${ }^{5}$ Furthermore, vitamin D can increase the activity of anti-inflammatory mediators and decrease the expression of proinflammatory cytokines. ${ }^{22}$ It is reported that patients with VDD have more severe disease than those with vitamin D insufficiency. ${ }^{20}$ However, based on our meta-analysis results,

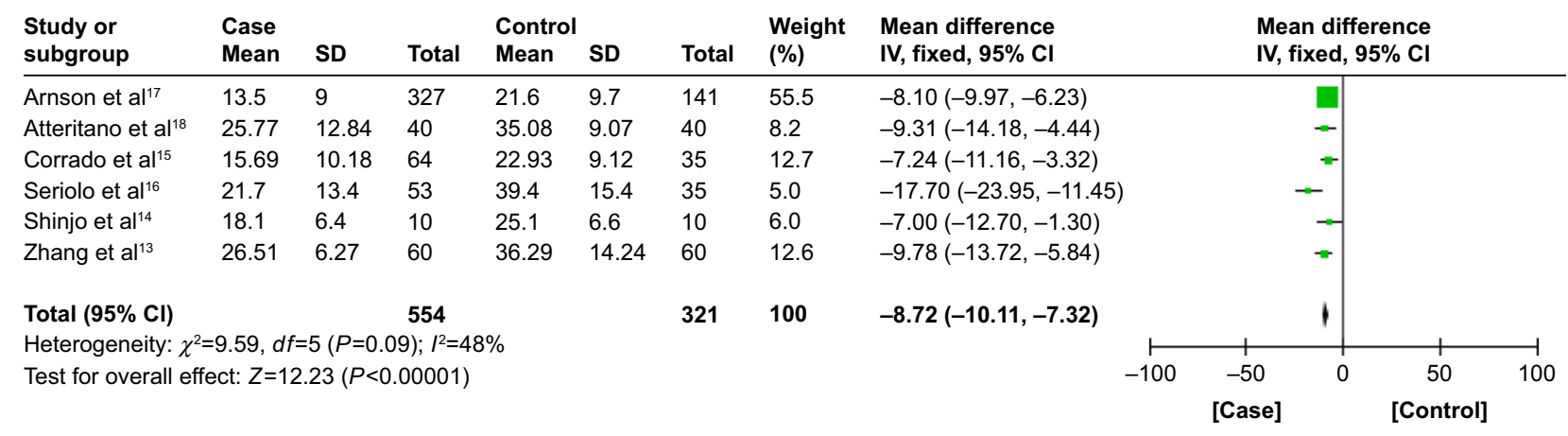

Figure 2 Meta-analysis of the vitamin D difference between case and control groups. Abbreviations: $\mathrm{Cl}$, confidence interval; IV, inverse variance; SD, standard deviation. 


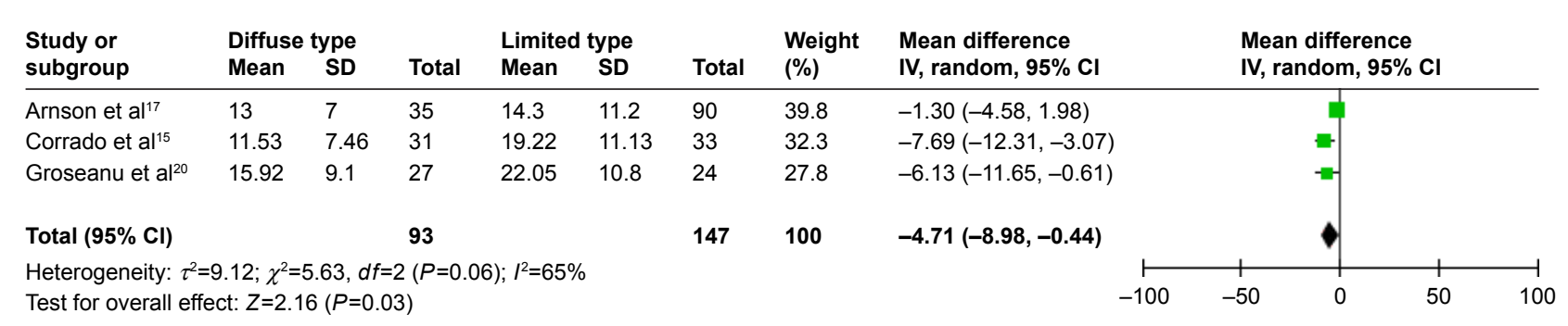

Figure 3 Meta-analysis of the vitamin D difference between diffused- and limited-type SSc patients.

Abbreviations: $\mathrm{Cl}$, confidence interval; IV, inverse variance; SD, standard deviation; SSc, systemic sclerosis.

A

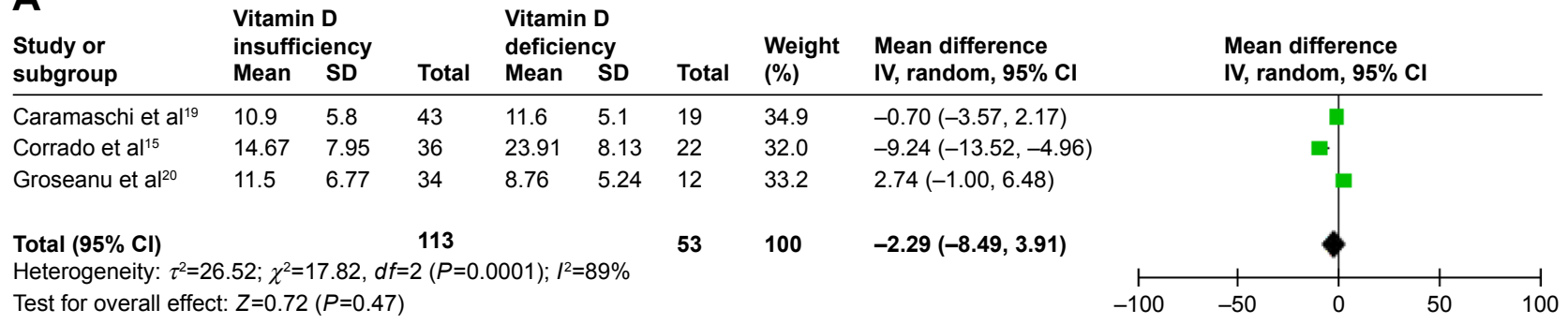

\section{B}

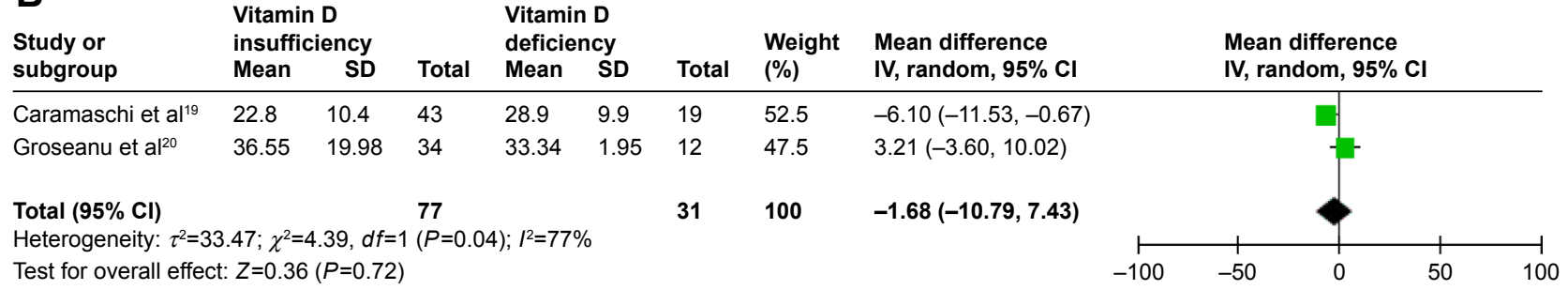

\section{C} Study or
subgroup

Vitamin D

Caramaschi et al $^{19}$ Groseanu et $\mathrm{al}^{20}$

insufficiency Vitamin D

Total $(95 \% \mathrm{Cl})$

Total events

Heterogeneity: $\chi^{2}=0.87, d f=1(P=0.35) ; l^{2}=0 \%$

deficiency

$5 \quad 19$

Events

\begin{tabular}{ll} 
Total & $\begin{array}{l}\text { Weight } \\
\text { (\%) }\end{array}$ \\
\hline 43 & 38.5 \\
34 & 61.5 \\
& \\
$\mathbf{7 7}$ & $\mathbf{1 0 0}$
\end{tabular}

Risk ratio

$\mathrm{M}-\mathrm{H}$, fixed, $95 \% \mathrm{Cl}$

Risk ratio

$1.41(0.53,3.76)$

$0.76(0.31,1.83)$

$1.01(0.53,1.93)$

Test for overall effect: $Z=0.03(P=0.98)$

23

23

$\mathrm{M}-\mathrm{H}$, fixed, $95 \% \mathrm{Cl}$

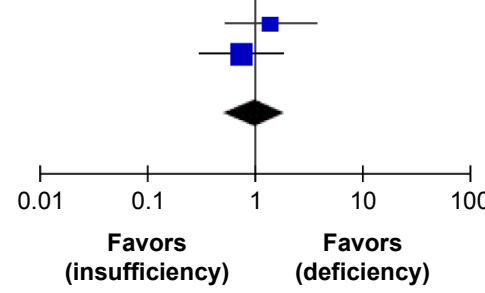

D

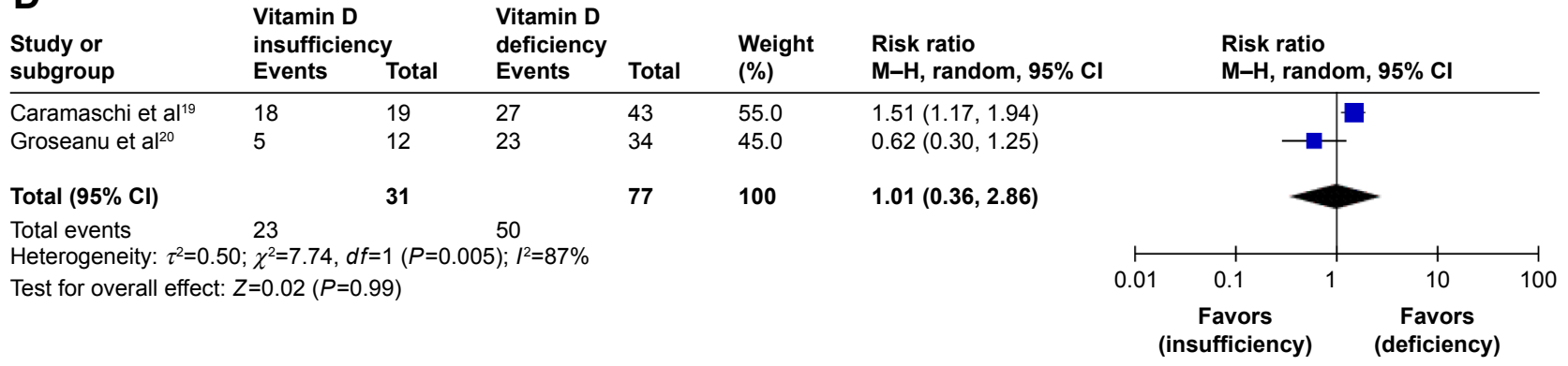

Figure 4 Meta-analysis of correlation of vitamin D level with Rodnan score $(\mathbf{A})$, systolic pulmonary pressure (B), gastrointestinal ulcer $(\mathbf{C})$ and pulmonary involvement in SSc patients (D).

Abbreviations: $\mathrm{Cl}$, confidence interval; IV, inverse variance; $\mathrm{M}-\mathrm{H}$, Mantel-Haenszel; SD, standard deviation; SSc, systemic sclerosis. 


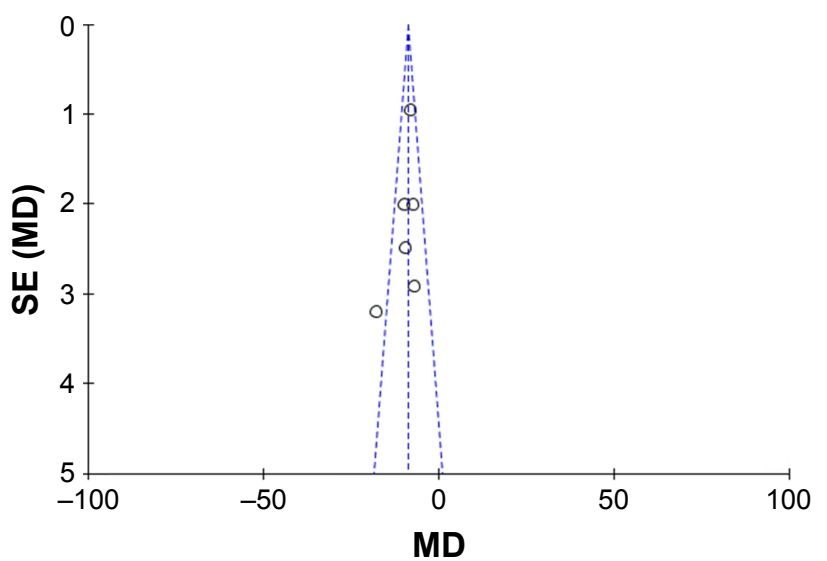

Figure 5 Funnel plots of included studies in the meta-analysis of the vitamin $D$ difference between case and control groups.

Abbreviations: MD, mean difference; SE, standard error.

no differences were found in the clinical characteristics including Rodnan score, gastrointestinal ulcer, pulmonary involvement or systolic arterial pressure between vitamin Dinsufficient and vitamin D-deficient SSc patients. Therefore, we deduce that a vitamin $\mathrm{D}$ decrease might not be an accelerating factor of SSc severity. Vacca et al reported that common vitamin D supplementation could not correct the lower vitamin D levels in SSc patients and that a higher dose is probably needed, especially in those with high inflammatory activity or severe disease. ${ }^{23}$ For SSc patients with or without vitamin D supplementation, Groseanu et al demonstrated that the vitamin D levels were higher for patients taking supplements, but the difference between the two groups did not reach statistical significance. However, patients with vitamin D supplementation developed less frequent gastrointestinal ulcers compared with those without, ${ }^{20}$ which suggests that the supplementation of vitamin D has some therapeutic effect in SSc treatment. As for the reason why standard doses of oral vitamin D supplements failed to correct the lower vitamin D status in SSc patients, reduced absorption capacity of thickening intestine is assumed to be the determinant for failure of oral treatment. ${ }^{24} \mathrm{~A}$ study carried out in Sweden showed that ultraviolet B (UVB) therapy was a more effective approach in increasing serum vitamin $D$ levels in SSc patients, suggesting that physical therapy might be a better treatment method for SSc patients. ${ }^{25}$ Considering that the above studies are based on a small sample size, precise studies with large-scale clinical trials are needed to assess the effect of vitamin D treatment on SSc outcomes and clarify whether VDD is a contributing factor to SSc.

\section{Limitations}

The primary concern in this meta-analysis is the limited sample size and number of included studies. Subsequently, we could not make a conclusion whether VDD is a risk factor or consequence of SSc. Lastly, most of the studies are retrospective rather than prospective. These limitations show that more prospective and high-quality studies with larger sample sizes are needed to further clarify the correlation of vitamin D with SSc, and especially whether vitamin D is a cause, accelerating factor or consequence of SSc.

\section{Conclusion}

SSc patients exhibited lower vitamin D levels compared with healthy controls. Vitamin D levels of diffused-type SSc patients were significantly lower than those in limitedtype SSc patients. The severity of clinical features was not associated with the extent of vitamin D deficit. Therefore, we hypothesize that SSc patients, especially diffused type, have lower vitamin D levels and that the decrease of vitamin D levels might not be an accelerating factor of SSc severity.

\section{Disclosure}

The authors report no conflicts of interest in this work.

\section{References}

1. Dankers W, Colin EM, van Hamburg JP, Lubberts E. Vitamin D in autoimmunity: molecular mechanisms and therapeutic potential. Front Immunol. 2017;7:697.

2. Arnson Y, Amital H, Shoenfeld Y. Vitamin D and autoimmunity: new aetiological and therapeutic considerations. Ann Rheum Dis. 2007; 66(9):1137-1142.

3. Holick MF, Chen TC, Lu Z, Sauter E. Vitamin D and skin physiology: a D-lightful story. J Bone Miner Res. 2007;22 (Suppl 2):V28-V33.

4. Upala S, Sanguankeo A. Low 25-hydroxyvitamin D levels are associated with vitiligo: a systematic review and meta-analysis. Photodermatol Photoimmunol Photomed. 2016;32(4):181-190.

5. Veldman CM, Cantorna MT, DeLuca HF. Expression of 1,25dihydroxyvitamin D3 receptor in the immune system. Arch Biochem Biophys. 2000;374(2):334-338.

6. Morgan JW, Kouttab N, Ford D, Maizel AL. Vitamin D-mediated gene regulation in phenotypically defined B cells subpopulations. Endocrinology. 2000;141(9):3225-3234.

7. Cutolo M. Further emergent evidence for the vitamin D endocrine system involvement in autoimmune rheumatic disease risk and prognosis. Ann Rheum Dis. 2013;72(4):473-475.

8. Elhai M, Avouac J, Kahan A, Allanore Y. Systemic sclerosis: recent insights. Joint Bone Spine. 2015;82(3):148-153.

9. Denton CP. Advances in pathogenesis and treatment of systemic sclerosis. Clin Med (Lond). 2016;16(1):55-60.

10. Giuggioli D, Colaci M, Cassone G, et al. Serum 25-OH vitamin D levels in systemic sclerosis: analysis of 140 patients and review of the literature. Clin Rheumatol. 2017;36(3):583-590.

11. Montabone E, Data V, Carignola R. Vitamin D status and quality of life in systemic sclerosis patients. J Clin Rheumatol. 2016;22(4):229-230.

12. Bivona G, Agnello L, Pivetti A, et al. Association between hypovitaminosis D and systemic sclerosis: true or fake? Clin Chim Acta. 2016; 458:115-119.

13. Zhang L, Duan Y, Zhang TP, et al. Association between the serum level of vitamin D and systemic sclerosis in a Chinese population: a case control study. Int J Rheum Dis. 2017;20(8):1002-1008.

14. Shinjo SK, Bonfá E, de Falco Caparbo V, Pereira RM. Low bone mass in juvenile onset sclerosis systemic: the possible role for 25hydroxyvitamin D insufficiency. Rheumatol Int. 2011;31(8):1075-1080. 
15. Corrado A, Colia R, Mele A, et al. Relationship between body mass composition, bone mineral density, skin fibrosis and $25(\mathrm{OH})$ vitamin D serum levels in systemic sclerosis. PLoS One. 2015;10(11): e0142748.

16. Seriolo B, Molfetta L, Cutolo M. Seasonal variations in serum levels of 25-hydroxyvitamin D in patients with systemic sclerosis. Clin Rheumatol. 2011;30(3):445-446.

17. Arnson Y, Amital H, Agmon-Levin N, et al. Serum 25-OH vitamin D concentrations are linked with various clinical aspects in patients with systemic sclerosis: a retrospective cohort study and review of the literature. Autoimmun Rev. 2011;10(8):490-494.

18. Atteritano M, Santoro D, Corallo G, et al. Skin involvement and pulmonary hypertension are associated with vitamin D insufficiency. Int J Mol Sci. 2016;17(12):2103.

19. Caramaschi P, Dalla Gassa A, Ruzzenente O, et al. Very low levels of vitamin D in systemic sclerosis patients. Clin Rheumatol. 2010; 29(12):1419-1425.

20. Groseanu L, Bojinca V, Gudu T, et al. Low vitamin D status in systemic sclerosis and the impact on disease phenotype. Eur J Rheumatol. 2016;3(2):50-55.
21. Yang CY, Leung PS, Adamopoulos IE, Gershwin ME. The implication of vitamin D and autoimmunity: a comprehensive review. Clin Rev Allergy Immunol. 2013;45(2):217-226.

22. Fernandes de Abreu DA, Eyles D, Féron F. Vitamin D, a neuroimmunomodulator: implications for neurodegenerative and autoimmune diseases. Psychoneuroendocrinology. 2009;34 (Suppl 1): S265-S277.

23. Vacca A, Cormier C, Piras M, Mathieu A, Kahan A, Allanore Y. Vitamin D deficiency and insufficiency in 2 independent cohorts of patients. J Rheumatol. 2009;36(9):1924-1929.

24. Robberecht E, Vandewalle S, Wehlou C, Kaufman JM, De Schepper J. Sunlight is an important determinant of vitamin D serum concentrations in cystic fibrosis. Eur J Clin Nutr. 2011;65(5):574-579.

25. Bogh MK, Gullstrand J, Svensson A, Ljunggren B, Dorkhan M. Narrowband ultraviolet B three times per week is more effective in treating vitamin D deficiency than 1600 IU oral vitamin D3 per day: a randomized clinical trial. Br J Dermatol. 2012;167(9):625-630.

\section{Publish your work in this journal}

Drug Design, Development and Therapy is an international, peerreviewed open-access journal that spans the spectrum of drug design and development through to clinical applications. Clinical outcomes, patient safety, and programs for the development and effective, safe, and sustained use of medicines are the features of the journal, which has also been accepted for indexing on PubMed Central. The manuscript management system is completely online and includes a very quick and fair peer-review system, which is all easy to use. Visit http://www.dovepress.com/testimonials.php to read real quotes from published authors.

Submit your manuscript here: http://www.dovepress.com/drug-design-development-and-therapy-journal 